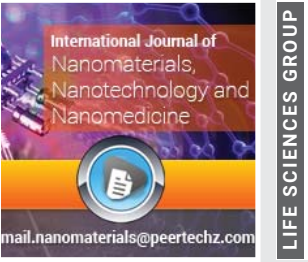

\section{Deduction of relativistic length variations based on tests using a Cryogenic Optical Resonator}

\section{Robert J Buenker*}

Department of C-Mathematics and Natural Sciences, University of Wuppertal, Gaussstr. 20, D-42097 Wuppertal, Germany

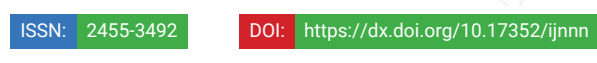

Received: 29 June, 2020

Accepted: 11 July, 2020

Published: 14 July, 2020

*Corresponding author: Robert J Buenker, Department of C-Mathematics and Natural Sciences, University of Wuppertal, Gaussstr 20, D-42097 Wuppertal, Germany, E-mail: rjbuenker@gmail.com

Keywords: Einstein's Symmetry Principle (ESP); Asymmetric time dilation; Absolute simultaneity; Clock-rate proportionality; Universal Time-Dilation Law (UTDL); Lorentz Transformation (LT); Relativistic Velocity Transformation (RVT); Newton-Voigt Transformation (NVT); Isotropic length expansion; Uniform scaling of physical properties; Amended version of the Relativity Principle (RP)

https://www.peertechz.com

Check for updates

\title{
Abstract
}

Experiments with the transverse Doppler effect have demonstrated that the wavelength of light increases with the speed of the source relative to the observer. The relativity principle implies that such a change cannot be detected by in situ measurements and this prediction has been verified by wavelength determinations carried out with a cavity resonator over an extended period of time during which the orbital speed of the Earth changes significantly. On this basis it can be concluded that the dimensions of the cavity resonator increase in direct proportion to the Doppler wavelength, and thus that isotropic length expansion occurs with relativistic time dilation, not the anisotropic Fitzgerald-Lorentz length contraction predicted by the special theory of relativity. The failure of the Lorentz space-time transformation to anticipate the length expansion effect is discussed and an alternative set of equations is introduced to eliminate the experimental contradiction in the existing theory.

\section{Introduction}

The experimental results reported in the work of Braxmaier, et al. [1], demonstrate that the speed of light $\mathrm{c}$ is independent of the state of motion of the observer to within a possible error of $\Delta \mathrm{c} / \mathrm{c}=(4.8 \pm 5.3) \times 10^{-12}$. This result represents a significant improvement over the original work of Kennedy and Thorndike [2], toward the goal of establishing the independence of the speed of light on the velocity of the laboratory, as postulated in Einstein's Special Theory of Relativity (STR) [3]. The experiments were carried out with an optical resonator over a period of 190 days during which time the orbital speed $\mathrm{v}$ of the Earth around the Sun changes by as much as one part in 30. If the in situ speed of light c (v) in the laboratory were dependent on $v\left(c=299792458 \mathrm{~ms}^{-1}\right)$, then the frequency of the resonator, given by $v_{\text {res }}=\mathrm{m} \mathrm{c}(\mathrm{v}) / 2 \mathrm{l}$ in the experiment (l is the length of the resonator and $\mathrm{m}$ is the constant mode number) would also not be constant, contrary to what was observed.

Yet we know that the frequency and wavelength of light waves do change when the light source is accelerated relative to the observer. Experiments with the transverse Doppler effect [4], have demonstrated that the wavelength of light varies in direct proportion to $\gamma(\mathrm{v})=\left(1-\mathrm{V}^{2} / \mathrm{c}^{2}\right)^{-0.5}$, where $\mathrm{v}$ is the speed of the light source relative to the laboratory. The corresponding light frequency decreases by the same factor [5], so that their product, the speed of light in free space, is independent of $\mathrm{v}$, in accord with Einstein's second postulate of STR [3]. Experiments carried out on airplanes [6] and satellites [7], have provided unequivocal evidence that the rates of atomic clocks slow down upon acceleration, but that the speed $\mathrm{v}$ that needs to be used to obtain the correct value of $\gamma$ in the above formula must be determined relative to a definite rest system, namely the frame of the Earth's non-rotating polar axis.

The question to be considered below is whether analogous changes in the lengths of objects occur when they are accelerated. The experimental situation is qualitatively different than for clock rates because there is no direct means of ascertaining whether the length of an object has varied during the course of its journey. By contrast, one only has to compare the roundtrip time shown on an accelerated clock with that of one left behind at its original location when they are brought together again to see if their rates have differed at any point. In other words, clocks have "memories," but measuring rods do not. 
In the absence of actual measurements, there has been wide reliance on theory in the form of the Fitzgerald-Lorentz length contraction (FLC) effect to simply predict the way lengths vary with velocity. The latter is derived from the Lorentz Transformation (LT) of STR [3], that has traditionally been looked upon as uniquely satisfying Einstein's postulates of relativity theory. This conclusion has recently [8-10], been shown to be incorrect, however, and an alternative spacetime transformation (Newton-Voigt Transformation, NVT) has been introduced in its place that has clear advantages over the LT in a number of areas, specifically in justifying the way in which the Global Positioning System (GPS) arrives at its distance determinations on the surface of the Earth. Within the framework of the NVT, it is possible to use the results of the cryogenic optical resonator experiments [1], to prove that the lengths of objects do vary with acceleration, but not in the manner predicted by the FLC.

\section{Inferences based on the Doppler effect and GPS metho- dology}

The main result of the cryogenic optical resonator experiments [1] is that, within the limits of experimental error, neither the in situ frequency nor the wavelength of light is found to change to any measurable degree as the Earth's orbit around the Sun varies between aphelion and perihelion. One should not lose sight of the fact that both quantities do change for an observer S located in the rest frame of the Sun. He must find that both the frequency $v(\mathrm{~S})$ and the wavelength $\lambda(\mathrm{S})$ of light in the optical resonator are constantly changing with the Earth's orbital speed v. Experiments with the transverse Doppler effect [4] have demonstrated that the wavelength of light varies in direct proportion to $\gamma(\mathrm{v})=\left(1-\mathrm{v}^{2} / \mathrm{c}^{2}\right)^{-0.5}$, where $\mathrm{v}$ is the speed of the light source relative to the laboratory. The corresponding light frequency decreases by the same factor [5], so that their product, the speed of light in free space, is independent of $\mathrm{v}$, in accord with Einstein's second postulate of STR [3] (note that Voigt [11], already invoked the light-speed constancy condition in 1887 to arrive at a relativistic space-transformation). The condition for resonance within the optical cavity in the above experiments [1] is maintained to the cited degree of accuracy for the entire period of 190 days. It therefore follows that the number of wavelengths $n$ in the cavity is always equal to $m / 2$, both for the observer E at rest on the Earth's surface and also for $\mathrm{S}$, since $\mathrm{n}$ is a relativistic invariant.

Since the wavelength of light $\lambda(S)$ is constantly varying for observer $S$ as the Earth makes its way around the Sun, however, this means that the length of the cavity $L(S)$ must have changed by the same proportion from his perspective over the same period of time. Specifically, if $S$ finds that the wavelength $\lambda(S)$ increases by a factor of $\mathrm{Q}$ as the Earth has accelerated between the aphelion and perihelion of its orbit, then the corresponding length $\mathrm{L}(\mathrm{S})$ of the cavity must have increased by $\mathrm{Q}$ based on his measurements. If $\mathrm{L}$ did not vary for $\mathrm{S}$, then he would have to see a steady departure from the condition of resonance in the cavity, contrary to the requirement that $\mathrm{n}$ be the same for all observers.

Because of the transverse Doppler effect [5], the frequency $v(S)$ measured in the rest frame of the Sun must have decreased by the same factor $\mathrm{Q}>1$ over the same period (after excluding gravitational effects, as also is done in the discussion that follows). The fact that observer $\mathrm{E}$ on the Earth's surface continues to measure the same value for $v_{\text {res }}$, i.e. $v(E)$, means that his laboratory clocks have slowed down. In other words, time dilation has occurred for these clocks as a result of the Earth's acceleration relative to the Sun. The argument given in the preceding paragraph shows that both $\lambda(\mathrm{S})$ and $\mathrm{L}(\mathrm{S})$ increase by the same factor $Q$ over this period of time. The conclusion is that time dilation is accompanied by isotropic length expansion in the above experiments [8-10].

The reason that both observers $\mathrm{S}$ and $\mathrm{E}$ continue to find that the speed of light has a constant value of c over the entire period of measurement is easily understood on this basis. Because of the time dilation, E measures shorter elapsed times $\mathrm{T}$ than does $\mathrm{S}$ by a factor of $\mathrm{Q}$ i.e. $\mathrm{T}(\mathrm{E})=\mathrm{Q}^{-1} \mathrm{~T}(\mathrm{~S})$. Because of the length expansion, E's meter stick is $Q$ times longer than $S$ 's. Therefore, E measures smaller distances $\mathrm{L}(\mathrm{E})$ travelled by the light by the same factor $Q$ than does $S$, i.e. $L(E)=Q^{-1} L(S)$. Therefore, the ratio of length traveled to time elapsed is exactly the same for both: $\mathrm{L}(\mathrm{S}) / \mathrm{T}(\mathrm{S})=\mathrm{L}(\mathrm{E}) / \mathrm{T}(\mathrm{E})=\mathrm{c}$. The cancellation effect for their respective time and distance measurements causes them to obtain the same value for the relative velocity of any two objects, not just for the speed of light relative to a given observer [12].

The fact that E's meter stick is larger than S's is also clearly consistent with the transverse Doppler effect $[4,5]$. He therefore measures a smaller value for the wavelength than does $S: \lambda(E)$ $=\mathrm{Q}^{-1} \lambda(\mathrm{S})$. Since the time dilation effect requires that $v(\mathrm{E})=\mathrm{Q}$ $v(S)$, it again follows that the measured speed of light is the same for both observers. Note that for other than transverse motion a correction has to be made for both $\lambda(\mathrm{S})$ and $v(\mathrm{~S})$ to reflect the fact that the source is moving relative to the waves it produces. The actual demonstration of the transverse Doppler effect [4], employs such a procedure. On this basis it is clear that light propagates isotropically for both observers $\mathrm{E}$ and $\mathrm{S}$, consistent with the Michelson-Morley experiment [13] and also with the prescriptions of STR [3].

The above conclusion is also supported by experience with the GPS methodology. A Gedanken experiment has been outlined in recent work [8] that considers how a rod changes its length when it is placed in orbit on a satellite. The elapsed time $\mathrm{T}$ for light to traverse a rod of length $\mathrm{L} \mathrm{m}$ is first measured on the satellite while it is still on the ground. This is done using a clock $\mathrm{P}$ that later is launched into orbit with the satellite and another (E) that stays behind on the Earth's surface. In both cases the result is $\mathrm{T}=\mathrm{c}^{-1} \mathrm{~L} \mathrm{~s}$, consistent with the modern definition of the meter as the distance traveled by light in $\mathrm{c}^{-1}$ s. The experiments with the optical resonator [1] indicate quite clearly that $\mathrm{P}$ will continue to find the elapsed time to be $\mathrm{T}(\mathrm{P})=$ $\mathrm{C}^{-1} \mathrm{~L} \mathrm{~s}$ once the satellite reaches orbit. Any other result would violate the Relativity Principle (RP), so this value for $T(P)$ is perfectly consistent with the light-speed constancy postulate of STR [3] as well.

The GPS technology employs a "pre-corrected" clock X on the satellite whose rate has been adjusted to compensate for

Citation: Buenker RJ (2020) Deduction of relativistic length variations based on tests using a Cryogenic Optical Resonator. Int J Nanomater Nanotechnol Nanomed 6(2): 016-020. DOI: https://dx.doi.org/10.17352/2455-3492.000035 
the effects of time dilation. If we exclude gravitational effects on the satellite clocks for the time being, this means that $\mathrm{X}$, while still on the ground, must run faster than $\mathrm{E}$ by a fixed ratio defined to be equal to $Q(Q>1)$. Therefore, when $X$ reaches its final orbit, it runs at exactly the same rate as clock $\mathrm{E}$ that remains at rest on the Earth's surface, and therefore $Q$ times faster than clock $\mathrm{P}$ at this point. As a result, the time registered on clock $\mathrm{X}$, while in orbit, for the light to traverse the rod is equal to $T(X)=T(E)=C^{-1} Q L$ s. Since the speed of light is also equal to $\mathrm{c}$ for the observer on the Earth's surface, it therefore follows that E now measures a value for the length of the rod to be $\mathrm{QL} \mathrm{m}$ as a consequence. Since the unit of distance has not changed on the ground, this can only mean the rod on the satellite, since $Q>1$, has increased in length as a result of its being shot into orbit. Furthermore, there is no reason to expect that this result will depend on the orientation of the rod since the rates of the clocks used in the above determination are clearly unaffected by this detail. The conclusion is therefore that relativistic time dilation is accompanied by isotropic length expansion, and by exactly the same amount as has been deduced above by combining the results of the transverse Doppler effect $[4,5]$ and cavity resonator experiments [1]

Although gravitational effects have been neglected in the above arguments, this is not essential in arriving at the final result. Because of the gravitational red shift [14], the rates of clocks increase with altitude above the Earth's surface. In actual practice [15], the GPS pre-corrected clock X must be slowed while on the ground in order for it to have the same rate as its counterpart E on the Earth's surface after reaching orbit. This is because the gravitational increase in rate is greater than that of opposite sign caused by time dilation. In previous work, the latter effect has been referred to as the gravitational scaling of units [16], as opposed to the "kinetic" scaling [17], that results because of acceleration effects.

In the following, it will be assumed that the uncompensated satellite clock $\mathrm{P}$ in orbit runs $\mathrm{S}$ times faster $(\mathrm{S}>1)$ than clock $\mathrm{E}$ on the Earth's surface because of the red shift, but $Q$ times slower due to time dilation as in the above discussion. This means that clock $\mathrm{X}$ in orbit must run slower than $\mathrm{P}$ by a factor of $\mathrm{Q} / \mathrm{S}(\mathrm{Q}<\mathrm{S})$ in order to have the same constant rate as clock $\mathrm{E}$. Consistent with the results of the cavity resonator experiments [1] and the RP, the elapsed time read on clock $\mathrm{P}$ for the light to traverse the rod when the satellite is in orbit is still $\mathrm{T}(\mathrm{P})=\mathrm{c}^{-1} \mathrm{~L} \mathrm{~s}$, the same value as before launch. Because of inclusion of the red shift, however, the corresponding value for clock $\mathrm{X}$ is now $\mathrm{T}(\mathrm{X})$ $=\mathrm{c}^{-1}(\mathrm{Q} / \mathrm{S}) \mathrm{L} \mathrm{s}=\mathrm{QL} / \mathrm{Sc} \mathrm{s}$, the same value as recorded on clock $\mathrm{E}$. But the speed of light on the satellite is also changed by virtue of the red shift. The ground observer finds that light travels there at the higher speed of Sc because of the increase in altitude [14]. As a result, he still finds the length of the rod to be $\mathrm{QL} \mathrm{m}$, the same value as found above when gravitational effects were ignored. The conclusion is that lengths are not affected by changes in altitude even though the rates of clocks are [16]. Isotropic length expansion accompanies time dilation, independent of the difference in gravitational potential separating the object and the observer.

\section{Failure of the lorentz transformation}

The discussion in the preceding section has been based squarely on Einstein's two postulates of STR [3] as well as experimental observations of the transverse Doppler effect [4-5] and the way clocks are affected by acceleration [6-7]. The results of the cryogenic optical resonator experiments [1] serve to reinforce the belief in these postulates, and thus by themselves do not require any change in the views of mainstream physicists about relativity theory. Yet the conclusion of isotropic length expansion that has been reached above runs completely contrary to the traditional view of how the spatial dimensions of objects vary with their state of motion. To understand how this situation came about, it is only necessary to see how Einstein [3] used his two postulates to derive the Lorentz Transformation (LT).

First of all, he realized that the two postulates were insufficient in themselves to uniquely specify the space-time transformation he was seeking. This had been pointed out earlier Lorentz [18]. Einstein decided that he could remove the uncertainty by invoking symmetry in the form of the Lorentz invariance condition for the coordinates of a moving object measured by two observers in relative motion with speed $\mathrm{v}(\Delta \mathrm{x}$, $\Delta \mathrm{x}^{\prime}$ etc. are intervals of distance traveled and corresponding elapsed time $\left.\Delta \mathrm{t}, \Delta \mathrm{t}^{\prime}\right)$

$$
\Delta \mathrm{x}^{2}+\Delta \mathrm{y}^{2}+\Delta \mathrm{z}^{2}-\mathrm{c}^{2} \Delta \mathrm{t}^{2}=0=\Delta \mathrm{x}^{\prime 2}+\Delta \mathrm{y}^{\prime 2}+\Delta \mathrm{z}^{\prime 2}-\mathrm{c}^{2} \Delta \mathrm{t}^{\prime 2}
$$

The resulting transformation led to many predictions which could not be tested experimentally at that time. One of the most prominent of these was that the longstanding belief in the principle of simultaneity of events for all observers had to be discarded, but there were also others, such as the prediction of the time-dilation phenomenon and Fitzgerald-Lorentz length contraction (FLC). Combining his two postulates with eq. (1) leads unambiguously to the LT:

$$
\begin{aligned}
& \Delta \mathrm{x}=\gamma(\mathrm{v})\left(\Delta \mathrm{x}^{\prime}+\mathrm{v} \Delta \mathrm{t}^{\prime}\right)(2 \mathrm{a}) \\
& \Delta \mathrm{t}=\mathrm{\gamma}(\mathrm{v})\left(\Delta \mathrm{t}^{\prime}+\mathrm{v} \mathrm{c}^{-2} \Delta \mathrm{x}^{\prime}\right)(2 \mathrm{~b}) \\
& \Delta \mathrm{y}=\Delta \mathrm{y}^{\prime}(2 \mathrm{c}) \\
& \Delta \mathrm{z}=\Delta \mathrm{z}^{\prime} .(2 \mathrm{~d})
\end{aligned}
$$

According to eq. (2b), the elapsed time $\Delta t$ measured by one observer would depend on both the corresponding time for the same event $\Delta t^{\prime}$ measured by another observer and the position of the latter, thereby eliminating absolute simultaneity as a law of physics.

What we know today from various experiments, in particular the experience with the GPS technology, however, is that this prediction of the LT is false. Observers in relative motion employ clocks that run at different rates, but if the elapsed times for two events are equal for one of them, they must also be equal for the other [8]. They simply disagree on the unit of time in which to express their measurements, not on whether the events were simultaneous or not. Clearly, there would be no point in employing a pre-corrected clock on the GPS satellites if events there did not occur at the same time

Citation: Buenker RJ (2020) Deduction of relativistic length variations based on tests using a Cryogenic Optical Resonator. Int J Nanomater Nanotechnol Nanomed 
for an observer on the Earth's surface. Hence, the success of the GPS industry in making distance determinations proves unequivocally that the LT does not qualify as a valid space-time transformation, despite the fact that it does satisfy Einstein's other two postulates.

It is therefore not surprising that his predictions about relativistic changes in lengths also prove to be false since they too are based directly on the LT. In this case, one uses the inverse of eq. (2a) to derive the FLC by setting $\Delta t=0$ and thereby obtaining $\Delta \mathrm{x}^{\prime}=\mathrm{y}(\mathrm{v}) \Delta \mathrm{x}$. On this basis it can be concluded that the length of an object $(\Delta \mathrm{x})$ moving with speed $\mathrm{v}$ along the $\mathrm{x}$ axis relative to one observer would appear to be contracted by a factor of $y(v)$ compared to the corresponding value $\left(\Delta \mathrm{x}^{\prime}\right)$ measured by a local observer traveling with it. Since it is physically impossible to distinguish one inertial system from another, however, there is a symmetry principle whereby each observer would think that it was the other's measuring rod that was contracted [19]. At the same time, the observers would agree completely on their measured values for distances in perpendicular directions to their line of motion because of eqs. $(2 c-d)$. These conclusions were in agreement with earlier inferences made by Fitzgerald and Lorentz [20-22], based on their interpretations of the Michelson-Morley experiment [13] and this added further credence to the derivation of the FLC from the LT. However, the experiments with a cryogenic optical resonator, GPS and the transverse Doppler effect discussed above prove that the Lorentz invariance condition of eq. (1) is not a property of the true relativistic space-time transformation. Distances expand isotropically as clocks slow down, rather than contract anisotropically as the FLC asserts.

A condition other than eq. (1) is therefore required in order to specify the desired space-time coordinate transformation. This is provided by the simultaneity condition, $\Delta \mathrm{t}=\Delta \mathrm{t}$ ' espoused by classical physicists $[8,9,23]$. As discussed elsewhere [8-10], this leads to the following set of equations, which is referred to below as the Newton-Voigt Transformation (NVT):

$$
\begin{aligned}
& \Delta x=\eta\left(\Delta \mathrm{x}^{\prime}+\mathrm{v} \Delta \mathrm{t}^{\prime}\right)(3 \mathrm{a}) \\
& \Delta \mathrm{y}=\eta \gamma^{-1} \Delta \mathrm{y}^{\prime},(3 \mathrm{~b}) \\
& \Delta \mathrm{z}=\eta \gamma^{-1} \Delta \mathrm{z}^{\prime},(3 \mathrm{c}) \\
& \Delta \mathrm{t}=\Delta \mathrm{t}^{\prime}, \text { (3d) }
\end{aligned}
$$

where $\eta=\left(1+\mathrm{v} \mathrm{c}^{-2} \Delta \mathrm{x}^{\prime} / \Delta \mathrm{t}^{\prime}\right)^{-1}$. Both of Einstein's postulates are satisfied thereby, but not the Lorentz invariance condition of eq. (1). Note that the same set of equations is obtained by multiplying both sides of the relativistic velocity transformation by $\Delta t=\Delta t^{\prime}[8,22]$. The key point to recognize is that the respective units of distance and time are assumed to be the same for both observers in these equations. In practical terms, this means that the observer on the GPS satellite has to base his timing measurements on the pre-corrected clock $\mathrm{X}$ so as to have agreement with the corresponding values read from the identical clock $\mathrm{E}$ on the Earth's surface. The units of time and distance are both $\mathrm{Q}=\gamma$ times greater on the satellite (if one corrects for gravitational effects) because of the kinetic scaling of units $[16,17]$. It is not possible to derive the latter relationships from the NVT directly, but there is nothing to keep one from using them consistently in eqs. (3a-d), i.e., the NVT is completely unchanged by converting between the set of "natural" units on the satellite and that used on the Earth's surface $[8,23]$.

There is a less complicated way to take account of the differences in the respective units employed in the two rest frames, however. One can simply include the conversion factor $Q^{\prime}=1 / Q$

explicitly in the corresponding equations as follows:

$$
\begin{aligned}
& \Delta \mathrm{x}=\eta\left(\Delta \mathrm{x}^{\prime}+\mathrm{v} \Delta \mathrm{t}^{\prime}\right) / \mathrm{Q}^{\prime}(4 \mathrm{a}) \\
& \Delta \mathrm{y}=\eta \Delta \mathrm{y}^{\prime} / \gamma \mathrm{Q}^{\prime},(4 \mathrm{~b}) \\
& \Delta \mathrm{z}=\eta \Delta \mathrm{z}^{\prime} / \gamma \mathrm{Q}^{\prime},(4 \mathrm{c}) \\
& \Delta \mathrm{t}=\Delta \mathrm{t}^{\prime} / \mathrm{Q}^{\prime} .(4 \mathrm{~d})
\end{aligned}
$$

\section{Conclusion}

The cryogenic optical resonator experiments [1], demonstrate in a quantitative manner that the in situ wavelength of light does not change with the laboratory's state of motion as it orbits the Sun over a period of many days. This study thus provides additional evidence for the Relativity Principle (RP) proposed by Galileo in the $17^{\text {th }}$ century and used by Einstein as his first postulate of relativity theory. We know from measurements of the transverse Doppler effect [4] that the wavelength does change for a stationary observer in the Sun's rest frame, however. The only way to reconcile these two observations is to assume that the wavelength actually does change in the cavity resonator over time, but that the reason this is not detected is because there is a proportional change in the lengths of all other objects in the laboratory's rest frame, including the resonator itself

Verification for this conclusion is found in the GPS methodology [8]. The length of a given rod on a satellite must appear to be the same when measured in situ after orbit has been achieved as it was before being launched from the Earth's surface. Yet the pre-corrected clock on the satellite, which runs at exactly the same rate as identical clocks on Earth and therefore faster than the uncompensated clock, must find that it takes more time for light to traverse the rod (after correcting for the gravitational red shift) when it is co-moving with the satellite than was measured on the Earth-bound clocks prior to launch. Since the speed of light is independent of the state of motion of the source, consistent with Einstein's second postulate and measurements of the transverse Doppler effect, it again follows that the length of the object has increased by virtue of its increased speed relative to its original position. The conclusion in both cases is that the periods of accelerated clocks increase in exactly the same proportion as the dimensions of co-moving rods. In short, time dilation is accompanied by isotropic length expansion. The units of time and distance in a given rest frame change in strict proportion as it undergoes acceleration. The scaling of these units is perfectly uniform, however, so that, consistent with the RP, it is impossible for a 
local observer to detect these changes based solely on his in situ measurements.

Physicists have consistently claimed that lengths of moving objects contract by varying amounts depending on their orientation to the observer. Further, they have argued that the effect is symmetric, that is, that observers can disagree as to which of two lengths is shorter, thereby denying the longstanding principle of objectivity or rationality of measurement. The reason for these deep-seated beliefs can easily be traced to the Lorentz Transformation (LT), the cornerstone of Einstein's STR [3]. It is easy to show that the LT is contradicted by experiment, however, particularly with regard to its prediction that events that occur simultaneously for one observer may occur at different times for another. Studies of atomic clocks on airplanes [6] and rockets [7] prove that their rates in different rest frames are always strictly proportional to one another. This excludes any possibility of observers disagreeing on whether two events occurred simultaneously, as a Gedanken experiment on GPS satellites clearly shows [8].

It is therefore not at all surprising that the LT is wrong about length contraction, especially when it is pointed out that there is an alternative transformation (NVT) that not only satisfies Einstein's two postulates but also rests squarely on the principle of the absolute simultaneity of events for all observers, regardless of their respective states of motion or positions in a gravitational field. The NVT is also consistent with time dilation and isotropic length expansion, and therefore with the optical resonator and Doppler experiments as well as with the GPS methodology. It therefore represents a significant advance in relativity theory, retaining all the proven successes of the LT while eliminating the latter's failure to satisfactorily predict the results of experiments that were impossible to carry out at the time it was first introduced.

\section{References}

1. Braxmaier C, Müller H, Pradl O, Mlynek J, Peters A, et al. (2002) Tests of Relativity Using a Cryogenic Optical Resonator. Phys Rev Lett 88: 010401. Link: https://bit.ly/2OpyfQV

2. Kennedy RJ, Thorndike EM (1932) Experimental Establishment of the Relativity of Time. Phys Rev 42: 400. Link: https://bit.ly/3iVPNSN

3. Einstein A (1905) On the Electrodynamics of Moving Bodies. Ann Physik 17: 891. Link: https://bit.ly/32b6oMB

4. Ives HE, Stilwell GR (1938) An Experimental Study of the Rate of a Moving Atomic Clock. J Opt Soc Am 28: 215-226. Link: https://bit.ly/2CwVmq1

5. Kuendig W (1963) Measurement of the Transverse Doppler Effect in an Accelerated System. Phys Rev 129: 2371. Link: https://bit.ly/3iUrzbH

6. Hafele JC, Keating RE (1972) Around-the-World Atomic Clocks: Predicted Relativistic Time Gains. Science 177: 166 -168. Link: https://bit.ly/3iV6NJ5

7. Vessot RFC, Levine MW (1979) A test of the equivalence principle using a space-borne clock. General Relativity and Gravitation 10: 181-204. Link: https://bit.ly/3eiCN6k
8. Buenker RJ (2008) The Global Positioning System and the Lorentz Transformation. Apeiron 15: 254. Link: https://bit.ly/3gTSLWd

9. Buenker RJ (2009) Simultaneity and the constancy of the speed of light: Normalization of space-time vectors in the Lorentz transformation. Apeiron 16: 96-146. Link: https://bit.ly/3emRyVP

10. Buenker RJ (2014) Relativity Contradictions Unveiled: Kinematics, Gravity and Light Refraction. Apeiron Montreal 55-56. Link: https://bit.ly/3fjrBHU

11. Voigt W (1887) Goett Nachr 41.

12. Buenker RJ (2015) On the Equality of Relative Velocities Between Two Objects for Observers in Different Rest Frames. Apeiron 20: 73-83. Link: https://bit.ly/2WdDtne

13. Michelson AA, Morley EW (1887) Am J Sci 34: 333.

14. Einstein A (1907) Über das Relativitätsprinzip und die aus demselben gezogenen Folgerungen. Jahrb Rad Elektr 4: 411. Link: https://bit.ly/2Wd5paN

15. Will CM (1993) Was Einstein Right?: Putting General Relativity to the Test Basic Books Inc. $2^{\text {nd }}$ Ed., New York, 272

16. Buenker RJ (2008) Gravitational and Kinetic Scaling of Physical Units. Apeiron 15: 382-413. Link: https://bit.ly/32bZyqg

17. Buenker RJ (2018) The Relativity Principle and the Kinetic Scaling of the Units of Energy, Time and Distance. Apeiron 20: 1-31. Link: https://bit.ly/3h2QUhQ

18. Lorentz HA, Versl K, Amsterdam 10: 793 Collected Papers. 5: 139.

19. Goldstein H (1950) Classical Mechanics, Addison-Wesley Publishing Co. Reading, Massachusetts 193. Link: https://bit.ly/2OfYAB0

20. Fitzgerald GF (1889) Science 13: 390.

21. Pais A (1982) in 'Subtle is the Lord. ' The Science and the Life of Albert Einstein, Oxford University Press, Oxford 122. Link: https://bit.ly/2DsB5m3

22. Lorentz HA, Versl K, Amsterdam Ak (1892) Collected Papers 4: 219.

23. Buenker RJ (2018) Stellar Aberration and Light-speed Constancy. Adv Theo Comp Phys 1: 1-4. Link: https://bit.ly/2AQIV86

Discover a bigger Impact and Visibility of your article publication with

Peertechz Publications

\section{Highlights}

* Signatory publisher of ORCID

* Signatory Publisher of DORA (San Francisco Declaration on Research Assessment)

* Articles archived in worlds' renowned service providers such as Portico, CNKI, AGRIS, TDNet, Base (Bielefeld University Library), CrossRef, Scilit, J-Gate etc.

* Journals indexed in ICMJE, SHERPA/ROMEO, Google Scholar etc.

* OAI-PMH (Open Archives Initiative Protocol for Metadata Harvesting)

* Dedicated Editorial Board for every journal

* Accurate and rapid peer-review process

* Increased citations of published articles through promotions

* Reduced timeline for article publication

Submit your articles and experience a new surge in publication services (https://www.peertechz.com/submission).

Peertechz journals wishes everlasting success in your every endeavours.

Copyright: @ 2020 Buenker RJ. This is an open-access article distributed under the terms of the Creative Commons Attribution License, which permits unrestricted use distribution, and reproduction in any medium, provided the original author and source are credited.

Citation: Buenker RJ (2020) Deduction of relativistic length variations based on tests using a Cryogenic Optical Resonator. Int J Nanomater Nanotechnol Nanomed 6(2): 016-020. DOI: https://dx.doi.org/10.17352/2455-3492.000035 